\title{
Un agonista del recettore della Somatostatina di tipo 3 (SSTR3) mostra effetti antitumorali in modelli sperimentali di tumori ipofisari non funzionanti
}

\author{
Giovanna Mantovani ${ }^{1} \cdot$ Federica Mangili $^{1}$
}

Pubblicato online: 9 luglio 2020

(c) The Author(s) 2020

Commento a:

A Somatostatin receptor subtype-3 (SST3) peptide agonist shows antitumor effects in experimental models of nonfunctioning pituitary tumors.

M.C. Vázquez-Borrego, V. Gupta, A. Ibáñez-Costa, M.D. Gahete, E. Venegas-Moreno, Á. Toledano-Delgado, D.A. Cano, C. Blanco-Acevedo, R. Ortega-Salas, M.A. Japón, A. Barrera-Martín, A. Vasiljevic, J. Hill, S. Zhang, H. Halem, J. Solivera, G. Raverot, M.A. Gálvez, A. Soto-Moreno, M. Paez-Pereda, M.D. Culler, J.P. Castaño, R.M. Luque. Clin Cancer Res (2019) 26(4):957-969

Gli analoghi della somatostatina (SSA) rappresentano il trattamento farmacologico d'elezione per alcuni tumori ipofisari neuroendocrini (PitNET) funzionanti, grazie alla loro capacità di normalizzare i livelli ormonali, ridurre la massa tumorale e attenuare la sintomatologia clinica. Gli SSA disponibili in clinica (octreotide, lanreotide e pasireotide) mediano la loro azione terapeutica principalmente attraverso il legame dei recettori della somatostatina di tipo 2 e 5 (SST2/5) [1]. Tuttavia, il sottogruppo di tumori ipofisari non funzionanti (NF-PitNET), che costituisce il 30\% di tutti i PitNETs, è per lo più resistente alle terapie convenzionali. Una spiegazione potrebbe risiedere nel fatto che i NF-PitNETs esprimono elevati livelli di altri sottotipi recettoriali, come SST3 [2].

Il lavoro oggetto di questo Commento è stato quello di identificare agonisti selettivi per SST3 e determinarne l'eventuale potenziale terapeutico, analizzando gli effetti sui

G. Mantovani

giovanna.mantovani@unimi.it

1 Unità di Endocrinologia, Fondazione IRCCS Ca' Granda Ospedale Maggiore Policlinico, Dipartimento di Scienze Cliniche e di Comunità, Università degli Studi di Milano, Milano, Italia segnali e sulla crescita tumorale in culture primarie in vitro e in modelli preclinici in vivo di NF-PitNETs.

Come primo passo, tramite l'utilizzo di appropriati strumenti, gli autori hanno indentificato due peptidi, BIM-355 e BIM-071, come i più specifici, selettivi e potenti, sulla base dell'affinità per SST3. Sono stati quindi studiati gli effetti di questi composti sulle vie di segnale intracellulare mediato dal recettore. Dai risultati è emerso che BIM-355 e BIM-071 inibiscono significativamente la vitalità cellulare in maniera dose-dipendente, la secrezione di cromogranina-A e aumentano l'apoptosi cellulare in culture primarie di NF-PitNETs. Tuttavia, nel $38 \%$ dei tumori non è stata evidenziata una riduzione della vitalità cellulare in risposta al trattamento, costituendo un sottogruppo "non responsivo". Per valutare la differenza di risposta emersa dai saggi di vitalità, sono stati analizzati i livelli di espressione proteica e di mRNA di tutti i sottotipi di SST (1/5). I dati hanno evidenziato una maggiore espressione di SST3 nel gruppo di NF-PitNETs "responsivo" al trattamento con questi agonisti selettivi, ma non sono state riportate differenze significative in merito all'espressione degli altri sottotipi di SST. Gli autori hanno quindi identificato le vie direttamente coinvolte nella trasmissione del segnale del recettore dopo stimolazione con BIM-355. I dati mostrano una riduzione dei livelli di fosforilazione di proteine coinvolte nelle tre principali vie del segnale: MAPK, PI3K-AKT/mTOR e JAK/STAT, nel gruppo di NF-PitNETs "responsivi". Al contrario, tali effetti non sono stati evidenziati nel gruppo dei tumori "non responsivi". Sono stati valutati poi gli effetti del silenziamento di SST3 sulla vitalità cellulare. Dalle analisi emerge che il silenziamento di SST3 abolisce la capacità dell'agonista di ridurre la vitalità cellulare in culture primarie di NF-PitNET.

Gli autori hanno infine testato gli effetti di BIM-355 sulla crescita tumorale in un modello murino pre-clinico di PitNET. Le risposte in vivo hanno confermato un'efficace ri- 
duzione dose-dipendente della massa tumorale, a partire da due settimane di trattamento.

In conclusione, l'insieme di questi risultati ha permesso l'identificazione di agonisti selettivi e funzionali di SST3, facendo emergere il potenziale terapeutico dell'attivazione di questo recettore in cellule di NF-PitNETs. Il presente studio mostra altresì un'importante rilevanza traslazionale indicando SST3 come possibile nuovo bersaglio per il trattamento farmacologico dei NF-PitNETs.

Ringraziamenti Open access funding provided by Università degli Studi di Milano within the CRUI-CARE Agreement.

Nota della casa editrice Springer Nature rimane neutrale in riguardo alle rivendicazioni giurisdizionali nelle mappe pubblicate e nelle affiliazioni istituzionali.

Open Access This article is licensed under a Creative Commons Attribution 4.0 International License, which permits use, sharing, adap- tation, distribution and reproduction in any medium or format, as long as you give appropriate credit to the original author(s) and the source, provide a link to the Creative Commons licence, and indicate if changes were made. The images or other third party material in this article are included in the article's Creative Commons licence, unless indicated otherwise in a credit line to the material. If material is not included in the article's Creative Commons licence and your intended use is not permitted by statutory regulation or exceeds the permitted use, you will need to obtain permission directly from the copyright holder. To view a copy of this licence, visit http://creativecommons.org/licenses/by/4.0/.

\section{Bibliografia}

1. Theodoropoulou M, Stalla GK (2013) Somatostatin receptors: from signaling to clinical practice. Front Neuroendocrinol 34:228-252

2. Hofland LJ, Feelders RA, de Herder WW, Lamberts SW (2010) Pituitary tumours: the sst/D2 receptors as molecular targets. Mol Cell Endocrinol 326(1-2):89-98 